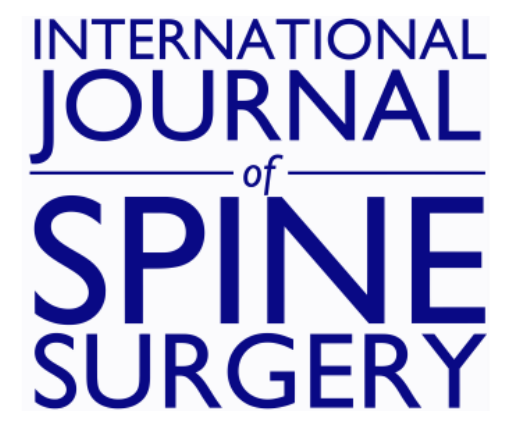

\title{
Computer Assisted Cobb Angle Measurements: A novel algorithm
}

Dean N. Papaliodis, Pierino G. Bonanni, Timothy T. Roberts, Khalid Hesham, Nicholas Richardson, Robert A. Cheney, James P. Lawrence, Allen L. Carl and William F. Lavelle

Int J Spine Surg 2017, 11 (3)

doi: https://doi.org/10.14444/4021

http://ijssurgery.com/content/11/3/21

This information is current as of April 26, 2023.

Email Alerts Receive free email-alerts when new articles cite this article. Sign up at:

http://ijssurgery.com/alerts

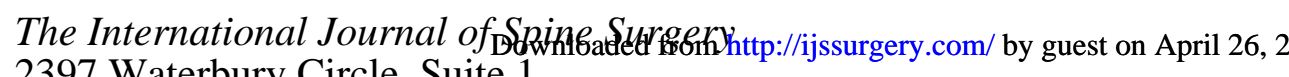
2397 Waterbury Circle, Suite 1,

Aurora, IL 60504, Phone: +1-630-375-1432

(C) 2017 ISASS. All Rights Reserved. 


\section{Computer Assisted Cobb Angle Measurements: A novel algorithm}

Dean N. Papaliodis, MD, ${ }^{1}$ Pierino G. Bonanni, PhD, ${ }^{2}$ Timothy T. Roberts, MD, ${ }^{3}$ Khalid Hesham, MD, ${ }^{1}$ Nicholas Richardson, MD, ${ }^{1}$ Robert A. Cheney, $M D,{ }^{4}$ James P. Lawrence, $M D, M B A,{ }^{5}$ Allen L. Carl, $M D, 6$ William F. Lavelle, $M D^{7}$

${ }^{1}$ Department of Orthopaedics, Albany Medical Center, Albany, NY, ${ }^{2}$ GE Global Research, Niskayuna, NY, ${ }^{3}$ Cleveland Clinic Center for Spine Health, Cleveland, OH, ${ }^{4}$ Bone \& Joint/ Capital Region Orthopaedics, Albany, NY, ${ }^{5}$ Bone \& Joint/ Capital Region Orthopaedics, Albany, NY, ${ }^{6}$ Department of Neurology, Albany Medical Center, Albany, NY, ${ }^{7 D e p a r t m e n t ~ o f ~ O r t h o p e d i c s, ~ S U N Y ~ U p s t a t e ~ M e d i c a l ~ U n i v e r s i t y, ~ S y r a c u s e, ~ N Y ~}$

\section{Abstract}

\section{Background}

The standard for evaluating scoliosis is PA radiographs using Cobb angle to measure curve magnitude. Newer PACS systems allow easier Cobb angle calculations, but have not improved inter/intra observer precision of measurement. Cobb angle and its progression are important to determine treatment; therefore, angle variability is not optimal. This study seeks to demonstrate that a performance equivalent to that achieved in the manual method is possible using a novel computer algorithm with limited user input. The authors compared Cobb angles from predetermined spinal levels in the average attending score versus the computer assisted approach.

\section{Methods}

Retrospective analysis of PA radiographs from 58 patients previously evaluated for scoliosis was collected. Predesignated spinal levels (e.g., T2-T10) were assigned for different curves and calculated by Cobb method. Four spine surgeons evaluated these Cobb angles. Their average scores were measured and compared to formulated values using the novel computer-based algorithm. Literature reports inter-observer reliability is 6.3-7.2degrees. Limits of accuracy were set at 5 degrees of average orthopedic surgeons' score.

\section{Results}

The computer-based algorithm calculated Cobb angles within 5 degrees of orthopedic surgeons' average with a standard deviation of 3.2 degrees. This result was based on a $95 \%$ confidence interval with $p$ values $<0.001$. The computer algorithm was plotted against average angle determined by the surgeons, with individual determinations and linear regression $\left(\mathrm{r}^{2}=0.90\right)$. The average difference between surgeons' measures and computer algorithm was 0.4 degrees $(\mathrm{SD}=3.2$ degrees, $\mathrm{n}=79)$. There was a tendency for the computer algorithm program to overestimate the angle at larger angles, but difference was small with $\mathrm{r}^{2}=0.09$.

\section{Conclusions}

Our study showed the novel computer based algorithm was an efficient and reliable method to assess scoliotic curvature in the coronal plane with the possibility of expediting clinic visits, ensuring reliability of calculation and decreasing patient exposure to radiation. Level of Evidence: III.

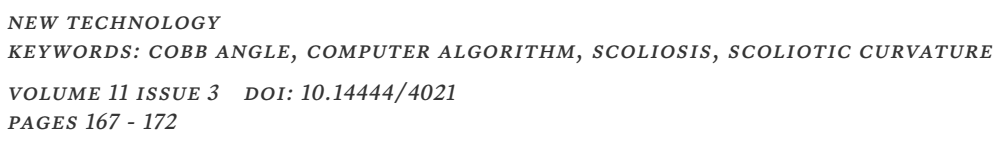

\section{Introduction}

Scoliosis is defined as a deviation in the coronal plane of the spine greater than 10 degrees. Although the deformity is three dimensional involving axial, sagittal and frontal planes, emphasis during diagnosis and progression is based on lateral deviation from the normal vertical line of the spine. The "gold stan- dard" for evaluating scoliosis is standing PA radiographs on large $36 \times 14$ inch cassettes utilizing the Cobb angle to measure the curve magnitude..$^{1-3}$

The Cobb angle is measured by drawing intersecting perpendicular lines from the cephalad end vertebra's superior surface and the caudad end vertebra's inferior surface, with their intersections forming the Cobb 
angle. Since the Cobb angle's introduction over fifty years ago, there has been little change on how physicians measure curve magnitude. ${ }^{4}$ The advent of digital capture and imaging modalities has had the potential to expand the possibility for scoliosis monitoring. New PACS systems do allow for easier Cobb angle measurements without the use of goniometers; however, it has not improved the inter- and intraobserver precision of the measurement. Although the calculation of scoliosis Cobb angle is a simple technique, there has been considerable interobserver variability (6.2-7.3 degrees) among physicians..$^{5-7}$ As a result of the large variability of the Cobb angle, a measurement difference of greater than 10 degrees needs to be documented between two radiographs taken at different points in time to receive 95\% statistical confidence that a true change in curve magnitude has occurred.

Zhang et al. ${ }^{8}$ developed a computer-aided system to reduce observer variability in radiographic scoliosis assessment. Their system semi-automatically measured the Cobb angle and vertebral rotation based on the Hough transform and snake model. Both the Cobb and rotation measurements resulted in average intra-observer and inter-observer errors less than 2 degrees and 3 degrees, respectively. They concluded that measurement variability is reduced using the developed system. In another study, Zhang et al. ${ }^{9}$ developed a computer-aided method to reduce the variability of Cobb angle measurement and wanted to determine if the method was sensitive to observer skill or experiences. This study found the variability of the Cobb measurement was reduced by using the computer-aided method. Dinesh Kumar et al. ${ }^{10}$ also described a novel application for content-based image retrieval of scoliosis images. They evaluated 30 curves on standing anteroposterior scoliosis images and found their system could retrieve correct sets of clinically matching images in the increasing order of distance. Wu et al. found the SurgimapSpine software measurement is an equivalent measuring tool compared to traditional manual in coronal plane Cobb angle. ${ }^{11}$

Since the Cobb angle and its progression are important components of determining scoliosis treatment, the variability of the angle is less than optimal. Im- provement in assessing the magnitude of scoliotic curves has the potential to reduce the necessary Xray exposure dose, expedite patient visits, and ensure the reliability of Cobb angle calculations.

This study adopts a computer-assisted approach proposed by Bonanni, ${ }^{12}$ in which estimates of the Cobb angle are derived from the overall structural curve of the spine and a related angle function, expressing the deviation from vertical as a continuous function of height along the spine. For purposes of this study, the Cobb angle estimates so derived are then refined by a subsequent matching step that seeks to align a set of derived reference lines to pre-designated vertebra surfaces in the image.

This study seeks to demonstrate that a performance equivalent to that achieved in the manual method is possible using a computer algorithm with limited user input. The authors compared Cobb angles from pre-determined spinal levels in the average attending score versus the computer-assisted approach. The hypothesis of this study is that the physician and computer-assisted estimates of the angle will be equivalent within 5 degrees.

\section{Materials and Methods}

Bonanni ${ }^{12}$ provides a method to extract Cobb angle estimates from coronal spine images based on a composite structural curve (CSC) and related angle function derived from left and right edges of the spine. As reported in that study, the estimates may deviate from manually derived Cobb angles if individual vertebrae are not aligned with the CSC. In this study, the Bonanni approach is employed to yield a set of initial estimates, but the estimates are subsequently refined by a visual matching step wherever a deviation is detected. A deviation is identified by a mismatch between a set of normal-to-CSC (i.e. perpendicular) reference lines and pre-designated vertebra endplates.

PA radiographs from 58 patients previously diagnosed with scoliosis from one academic center were collected with removal of patient identifiers. The patients presented in a consecutive fashion in the spine clinic to mirror what might be expected on average 
over a defined time period. Pre-designated spinal levels (T2-T10) were assigned for different curves to be calculated by the Cobb method. Utilizing the standard Cobb angle measurement calculation previously described, which involves physician interpretation and measurement, four trained orthopedic spine surgeons calculated the Cobb angles. Their average score was measured and compared to the formulated values using the computer assisted algorithm. Although the documented inter-observer variability is 6.3-7.2 degrees, we set our limits of accuracy to be within 5 degrees of the average surgeon score.

Expanding on the earlier description, the computerassisted image processing and curvature measurement methodology employed in this study consisted of an image enhancement step followed by a curvature measurement step deriving from the approach reported by Bonanni. ${ }^{12}$ The objective of enhancement was local adjustment of brightness and contrast to reveal the maximum anatomical detail within the dynamic range afforded by the display system. X-ray absorption varies with composition and thickness of intervening tissue, with higher densities and thicknesses resulting in greater attenuation. As these properties vary along the length of the spine, exposure settings optimized for one region may not be optimal for other regions. With conventional imaging equipment, this is evidenced at the light box or computer monitor by thoracic and lumbar regions typically appearing bright with the cervical region appearing dark.

An enhancement scheme was employed that normalized luminosity ranges so that all regions of the spine were maximally visible at a given display brightness and contrast setting. As illustrated in Figure 1, this was achieved by computing localized mean brightness at key points in the original (left) image and using these to construct a localized brightness map (middle image). In the enhanced (right) image, brightness values reflected offsets with respect to this map, rather than their original, absolute brightness.

Implemented for this study, the adjustment was realized through an image processing algorithm aided by user input. No morphological alteration of the image features was performed, as any alteration might im- pact the subsequent curvature measurement. The brightness mapping was an iterative process that combined the initial user input, analysis of the image brightness variation, re-mapping of brightness values, and subsequent feedback from the user on the result. This process repeated until an optimum mapping was obtained.

After enhancement, a second digital processing step targeted a measurement of the lateral curvature of the spine that could be correlated to Cobb angles. The curvature measurement technique used in this study, as prescribed by Bonanni, ${ }^{12}$ employed mouse input to select points defining left and right edges of the spine. From these data, smooth, order-5 polynomial fits were obtained and the resulting left and right edge curves averaged to derive the CSC. A series of normals describing the angular orientation of vertebrae were computed and overlaid on the digitally enhanced image. Further refinement of these normals was then performed by matching to the vertebra surface transitions at the pre-designated spinal levels. An example of this image processed by the computer algorithm is shown in Figure 2. All steps of the algorithm were implemented using custom software developed in the MATLAB programming environment (MATLAB and Image Processing Toolbox, Release 2012b, The MathWorks, Inc., Natick, MA).

\section{Statistical Methods}

With the 58 images (some with multiple curves) evaluated by both surgeons and the algorithm, this study
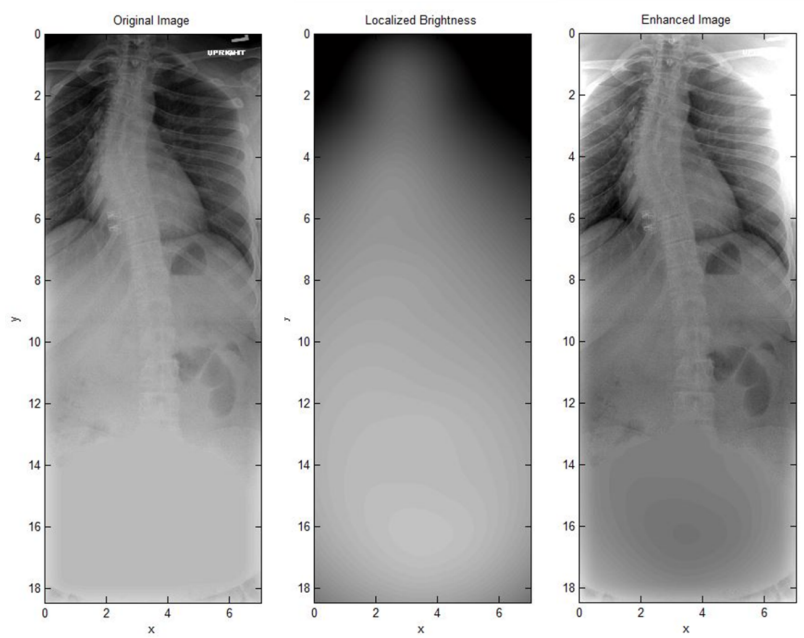

Fig. 1. Original, localized brightness, and enhanced images prior to curvature measurements. 
had a $93 \%$ power to yield a significant result using a 2 tailed alpha of 0.05 to detect an overall difference as large as 2.5 degrees. Data and statistical analysis was performed using Statistica (Statistic v6, StatSoft Co, Tulsa, OK). Cobb angles measured by the surgeons were averaged and compared to measurements of the same angle by the computer-assisted method using linear regression and the methodology of Bland and Altman which is useful for comparing two methods of measurement. ${ }^{13}$ Overall bias and bias as a function of the angle were assessed. The statistical significance was set at $\mathrm{p}<0.001$ with a $95 \%$ confidence interval.

\section{Results}

Our results indicated the computer based algorithm was able to calculate Cobb angles within 5 degrees of the trained orthopedic surgeons' average with a standard deviation of 3.2 degrees. This result is based on a $95 \%$ confidence interval with $p$ values $<0.001$. The computer algorithm was plotted against the average angle determined by the surgeons, with individual determinations and linear regression $\left(\mathrm{r}^{2}=0.90\right)$ (Figure 3). The overall surgeons' reliability values are presented in Table 1 with the surgeons' inter-rater reliability classified as "good." For qualitative purposes, the inter-observer correlation of 0.0 to 0.25 reflects absent to poor, $0.25-0.49=$ low, $0.50-0.69=$
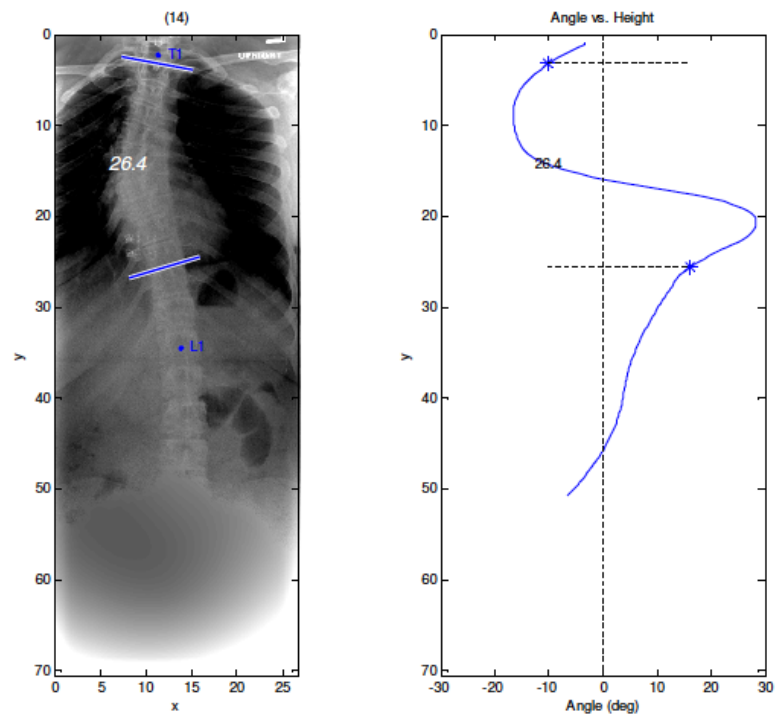

Fig. 2. (A) Computer based calculation of Cobb angle based on pre-designated spinal levels. (B) Surgeons' average calculation on this patient was 23.6 degrees with a standard deviation of 2.8 degrees. fair $/$ moderate, $0.70-0.89=$ good, and 0.90-1.0 excellent correlations.

The Bland-Altman analysis showed that the bias indicated by the line is small, and the average difference between the surgeons' measures and the computer algorithm was 0.4 degrees $(\mathrm{SD}=3.2$ degrees, $\mathrm{n}=79$ ). There was a tendency for the computer algorithm program based method $(\mathrm{PB})$ to overestimate the angle at larger angles, but the difference is small with $\mathrm{r}^{2}=0.09$ (Figure 4).

\section{Discussion}

Scoliosis is a common spinal pathology affecting adolescents with the Cobb angle used for quantitative assessment of the lateral curvature of the spine. Cobb angle measurement is standard for diagnosis and follow-up of patients with scoliosis. ${ }^{5}$ While the calculation is a simple technique, there has been considerable inter-observer variability of 6.2-7.3 degrees

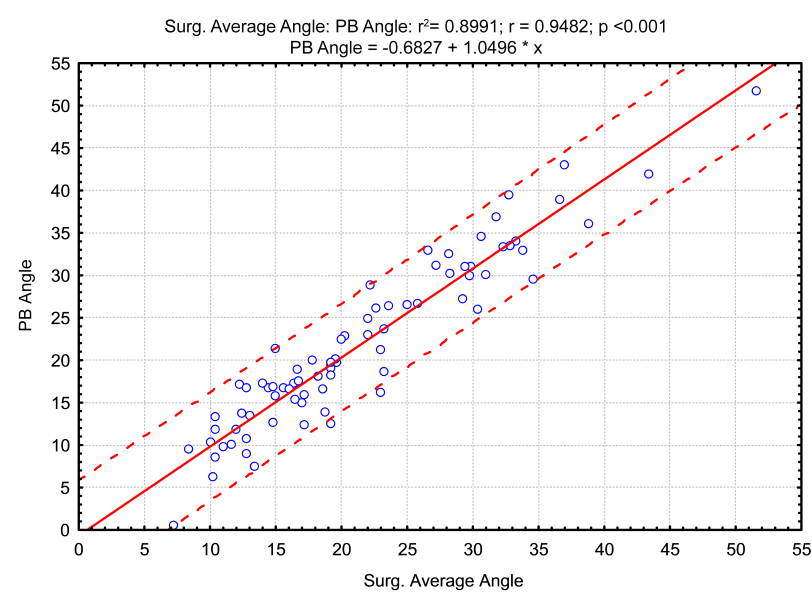

Fig. 3. Computer algorithm program-based (PB) determined angle plotted against the determined surgeons' average angle. Individual determinations are indicated by the symbol 0 ; solid line is the linear regression $\left(r^{2}=0.90\right)$ and dotted lines are the $95 \%$ prediction intervals.

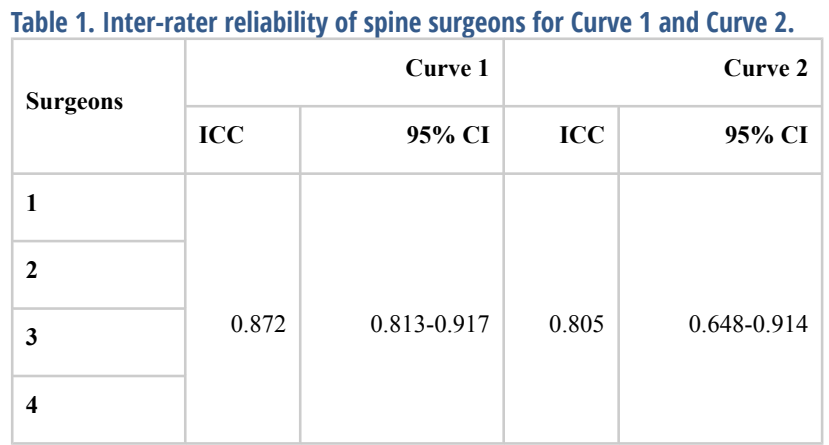

ICC: Interclass Correlation Coefficient; Cl: Confidence Interval. 
among practitioners. ${ }^{5-7}$ The computer based algorithm in our study proved to be as accurate as the orthopedic surgeons' average within 5 degrees of variability. While some studies have reported that digital methods for assessing Cobb angles increased the reliability of calculations, ${ }^{14,15}$ other studies have not found significant differences between manual and digital approaches. ${ }^{16}$ Supported by our statistical analysis, our computer based method was able to produce a reliable calculation with minimal bias as shown in Figure 4.

Korvessis and Stamatakis ${ }^{17}$ created two mathematical formulas that provided accurate Cobb angle with the use of only the scoliometer. They were able to predict the Cobb angle with a deviation of 5.63 degrees for thoracic curves and 5.79 degrees for lumbar curves. They also believed using their method would reduce the cost of school screening programs, overdiagnosis and unnecessary exposure to radiation. Zhang et al. ${ }^{9}$ used a fussy Hough transform technique to develop a computer-aided method to measure Cobb angle automatically. Their method showed an intra-observer and inter-observer error of less than 3 degrees for the Cobb angle. They believed their method could help surgeons measure the Cobb angle more reliably during scoliosis clinics. Stokes et al. ${ }^{18}$ sought to determine the reliability of a computerassisted measurement protocol for evaluating Cobb angle and the King et al classification. The computer program identified curves, calculated Cobb angles and generated the King et al. classification automatically. It analyzed the coordinates of the points

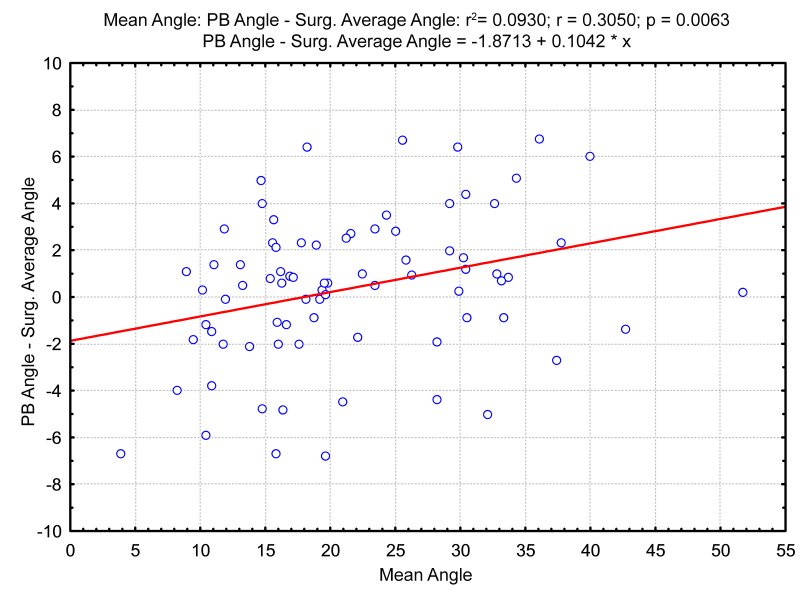

Fig. 4. Bland-Altman Analysis. Difference in angles measured by the two techniques versus the mean angle. marked by evaluators. The reliability was 2 degrees for thoracic and lumbar curves. The classification was more reliable than achieved by unassisted observers evaluating the same radiographs.

One limitation associated with the methodology employed in this study was the pre-designation of spinal levels. For instance, if one surgeon believes that the extreme spinal levels are from T1 to T10, and another believes the angle should be calculated from $\mathrm{T} 2$ to $\mathrm{T} 9$, then there would be variability in the measured angle. As implemented for this study, the methodology is limited by the visual acuity and judgment of the practitioner in designating spinal levels.

Potential future applications of this software hopefully include expediting patient visits by decreasing the amount of time needed to calculate measurements. However, the time for measurement would depend on many variables; and, we did not evaluate if the new method is more time efficient than the conventional Cobb technique in this study. Additionally, with poor quality images, it may be difficult for the practitioner to calculate the corresponding Cobb angles. Further studies and subgroup analyses would need to be performed for larger Cobb angles to further define the $r^{2}$ value. More research is needed to analyze if utilizing the computer based algorithm has the potential to decrease radiation exposure in adolescents who require multiple x-rays to attain a quality image for manual calculation. Additionally, the potential exists in fully automating this algorithm; thus, entirely removing user variability and standardizing results. In conclusion, our study showed that the novel computer-based algorithm was efficient and reliable in assessing scoliotic curvature in the coronal plane.

\section{References}

1. Cassar-Pullicino VN, Eisenstein SM. Imaging in scoliosis: what, why and how? Clin Radiol. $2002 \mathrm{Jul}$; 57(7): 543-62.

2. Dempsey M, Wilkes D, Moody M. Imaging Studies. In: Herring J. (ed.) Tachdjian's Pediatric Orthopaedics, $4^{\text {th }}$ Edition. Philadelphia: Saunders/Elsevier, 2007. 
3. Morrissey RT, Weinstein SL. Lovell and Winter's Pediatric Orthopaedics, $6^{\text {th }}$ Edition, Philadelphia: Lippincott, William \& Wilkins, 2006.

4. Lenke LG, Betz RR, Harms J, Bridwell KH, Clements DH, Lowe TG, Blanke K. Adolescent idiopathic scoliosis: a new classification to determine extent of spinal arthrodesis. J Bone Joint Surg Am. 2001 Aug; 83-A(8): 1169-81.

5. Langensiepen $S$, Semler O, Sobottke R, Fricke O, Franklin J, Schönau E, Eysel P. Measuring procedures to determine the Cobb angle in idiopathic scoliosis: a systematic review. Eur Spine J. 2013

Nov;22(11):2360-71.

6. Ricart PA, Andres TM, Apazidis A, Errico TJ, Trobisch PD. Validity of Cobb angle measurements using digitally photographed radiographs. Spine J. 2011 Oct;11(10):942-6.

7. Segev E, Hemo Y, Wientroub S, Ovadia D, Fishkin M, Steinberg DM, Hayek S. Intra- and interobserver reliability analysis of digital radiographic measurements for pediatric orthopedic parameters using a novel PACS integrated computer software program. J Child Orthop. 2010 Aug;4(4):331-41. 8. Zhang J, Lou E, Hill DL, Raso JV, Wang Y, Le $\mathrm{LH}$, Shi X. Computer-aided assessment of scoliosis on posteroanterior radiographs. Med Biol Eng Comput. 2010 Feb; 48(2): 185-95.

9. Zhang J, Lou E, Shi X, Wang Y, Hill Dl, Raso JV, Le LH, Lv L. A Computer-aided Cobb angle measurement method and its reliability. J Spinal Disord Tech. 2010 Aug; 23(6): 383-7.

10. Dinesh Kumar VP, Thomas T, Menon KV. Content-based image retrieval of spine radiographs with scoliosis. J Spinal Disord Tech. 2009 Jun;22(4): 284-9.

11. Wu W, Liang J, Du Y, Tan X, Xiang X, Wang W, $\mathrm{Ru}$ N, Le J. Reliability and reproducibility analysis of the Cobb angle and assessing sagittal plane by computer-assisted and manual measurement tools. BMC Musculoskelet Disord. 2014 Feb 6;15:33.

12. Bonanni PG. Contour and angle-function based scoliosis monitoring: relaxing the requirement on image quality in the measurement of spinal curvature.
Int J Spine Surg. 2017 June; 11(3):173-182.

13. Vrotovec T, Pernus F, Likar B. A review of methods for quantitative evaluation of spinal curvature. Eur Spine J. 2009 May; 18(5):593-607.

14. Shea KG, Stevens PM, Nelson M, Smith JT, Masters KS, Yandow S. A comparison of manual versus computer assisted radiographic measurement: Intraobserver measurement variability for Cobb angles. Spine. 1998 Mar 1;23(5):551-5.

15. Kuklo TR, Potter BK, Schroeder TM, O'Brien MF. Comparison of manual and digital measurements in adolescent idiopathic scoliosis. Spine. 2006 May 15; 31(11):1240-6.

16. Gstoettner M, Sekyra K, Walochnik N, Winter $\mathrm{P}$, Wachter R, Bach CM. Inter- and Intraobserver reliability assessment of the Cobb angle: manual versus digital measurement tools. Eur Spine J. 2007 Oct; 16(10):1587-92 .

17. Korovessis PG, Stamatakis MV. Prediction of scoliotic Cobb angle with the use of the scoliometer. Spine. 1996 Jul 15;21(14):1661-6.

18. Stokes IA, Aronsson DD. Computer-assisted algorithms improve reliability of King classification and Cobb angle measurement of scoliosis. Spine. 2006 Mar 15; 31(6) 665-70.

\section{Disclosures \& COI}

The authors declare no relevant financial disclosures or conflicts of interest.

\section{Corresponding Author}

Pierino Bonanni, PhD. GE Global Research, 1 Research Circle, Niskayuna, NY 12309. bonanni@ge.com.

Published 30 June 2017.

This manuscript is generously published free of charge by ISASS, the International Society for the Advancement of Spine Surgery. Copyright @ 2017 ISASS. To see more or order reprints or permissions, see http://ijssurgery.com. 